Journal of

Cardiology and Vascular Medicine

\title{
Oxidative Stress Parameters in Chronic Obstructive Pulmonary Disease with and without Pulmonary Hypertension
}

\section{Muntecep Asker, $\mathrm{MD}^{1}$, Selvi Asker $\mathrm{MD}^{2,{ }^{*}}$, Eren Sarikaya $\mathrm{MD}^{3}$}

${ }^{1}$ Department of Cardiology, Van Yuzuncu Yil University Medical Faculty, Van, Turkey ${ }^{2}$ Department of Chest Diseases, Yuzuncu Yil University Medical Faculty, Van, Turkey

${ }^{3}$ Department of Chemistry, Van Yuzuncu Yil University, Faculty of Science, Van, Turkey

${ }^{\star}$ Corresponding author: Selvi Asker, MD: Department of Chest Diseases, Yuzuncu Yil University School of Medicine, 65000 Van, Turkey, Tel: +905052514704, Fax: +904322121954; E-mail: selviasker@yahoo.com

Received Date: January 03, 2019 Accepted Date: January 21, 2020 Published Date: January 23, 2020

Citation: Muntecep Asker (2020) Oxidative Stress Parameters in Chronic Obstructive Pulmonary Disease with and without Pulmonary Hypertension. J Cardio Vasc Med 6: 1-7.

\begin{abstract}
Objective: The current study aimed to investigate whether levels of oxidative stress indicators, were altered in chronic pulmonary obstructive disease patients with and without pulmonary hypertension and to examine if oxidative stress markers are correlated with demographic, hematological, biochemical and cardiorespiratory variables.

Methods: Thirty chronic pulmonary obstructive disease patients ( 9 women, 21 men) and 30 healthy controls (20 women, 10 men) were included. Of the 30 patients, 18 (60\%) had been diagnosed with pulmonary hypertension. Demographic features, oxygen saturation, hematological, biochemical markers and oxidative stress indicators (catalase activity, glutathione and malondialdehyde levels) were measured and compared in not only chronic pulmonary obstructive disease and control groups, but also in chronic pulmonary obstructive disease patients with and without pulmonary hypertension.

Results: In chronic pulmonary obstructive disease patients, serum levels of malondialdehyde were increased; but concentrations of glutathione and catalase were significantly lower. Notably, chronic pulmonary obstructive disease patients with and without pulmonary hypertension did not exhibit any remarkable difference in terms of levels of oxidative stress indicators. An inverse correlation was detected between levels of glutathione and malondialdehyde.

Conclusions: To conclude, results of the current study support the roles of inflammation and oxidative stress in chronic pulmonary obstructive disease, but no remarkable alterations in oxidative stress indicators could be identified in chronic pulmonary obstructive disease patients with pulmonary hypertension. Therefore, further trials on larger series are required to elucidate the role of oxidative stress and to assess the effects of antioxidants in chronic pulmonary obstructive disease and pulmonary hypertension.
\end{abstract}

(C)2020 The Authors. Published by the JScholar under the terms of the Creative Commons Attribution License http://creativecommons.org/licenses/ by/3.0/, which permits unrestricted use, provided the original author and source are credited. 


\section{Introduction}

Chronic obstructive pulmonary disease (COPD) is an incurable but preventable respiratory disease with a high prevalence that is on the way to becoming one of the most common causes of death worldwide $[1,2]$. It is characterized by persistent progressive airflow limitation and hyperinflation enhanced by systemic and respiratory chronic inflammation. Tobacco smoke and airborne particulate matter may elicit the production and release of oxidative stressors in the airways [2,3]. The pathophysiology underlying COPD has not been completely understood yet. Products of oxidative stress may facilitate a series of inflammatory reactions leading to the pathogenesis of COPD [4].

Oxidative stress occurs when free radicals and other reactive species overwhelm the availability of antioxidants. Reactive oxygen species, reactive nitrogen species, and their counterpart antioxidant agents are essential for physiological signalling and host defense, as well as for the evolution and persistence of inflammation. When their normal steady-state is disturbed, imbalances between oxidants and antioxidants may provoke pathological reactions causing a range of nonrespiratory and respiratory diseases, particularly COPD [4].

The role of oxidative stress in COPD has been well established, but the occasion for pulmonary hypertension (PHT) co-existing with COPD is still under debate $[5,6]$. Pulmonary hypertension may occur as a complication of COPD and is linked with a negative prognosis $[7,8]$. Pulmonary vascular remodelling and vasoconstriction due to hypoxia contribute to the pathogenesis of PHT in the setting of COPD [5]. In animal models of PHT, increased pulmonary arterial pressure was related with increased oxidative stress [9]. Moreover, pulmonary oxidant/ antioxidant balance has been investigated in various forms of PHT and reduced antioxidant enzyme activity was detected in patients with severe PHT [9]. Joppa et al. have demonstrated the imbalance between oxidative stress and antioxidants in COPD patients and alterations in antioxidant levels of COPD patients with mild-moderate PHT were similar to those of patients without PHT [5].

In spite of these data, the relationship between oxidative stress in COPD patients with and without PHT has not been fully elucidated yet. The current study was performed in order to evaluate the alterations of oxidative stress markers, catalase, glutathione, and malondialdehyde in COPD patients with and without PHT. In addition, any possible correlations of these oxidative stress markers with demographic, clinical and biochemical variables were investigated.

\section{Method}

Study Design: This cross-sectional, clinical study was performed in the chest diseases department of a university hospital after the approval of the local Institutional Review Board. Written informed consent was obtained from all participants and the study was carried out between June 2013 and August 2013 in accordance with the principles of the Helsinki Declaration of 1975, as revised in 2000 .

Thirty COPD patients ( 9 women, 21 men) and 30 healthy controls (20 women, 10 men) were included. Of the 30 COPD patients, 18 (60\%) had been diagnosed with PHT. Demographic features of patients, as well as oxygen saturation, left ventricular ejection fraction measured by echocardiography, serum levels of haemoglobin ( $\mathrm{Hb})$, hematocrit (Hct), low-density lipoproteins (LDL), triglycerides (TG), high-density lipoproteins (HDL), cholesterol, C-reactive protein (CRP), fibrin degradation product (D-dimer), catalase (CAT), glutathione (GSH), and malondialdehyde (MDA) were measured. Chronic obstructive pulmonary disease patients with and without PHT and controls were compared in terms of these aforementioned demographic, hematological, biochemical and cardiorespiratory variables.

Exclusion criteria were composed of infectious diseases, acute respiratory distress, obstructive sleep apnea, pulmonary embolism, heart failure, malignancy, autoimmune disorders, recent surgery, endocrine, hepatic or renal diseases, interstitial lung disease and left ventricular failure in the last 3 months prior to the study. Besides the absence of the previous diagnosis of the studied diseases were excluded from the study. The control group comprised healthy adult volunteers with no history of smoking.

The diagnosis of COPD was established with respect to the guidelines of the American Thoracic Society [10]. Pulmonary hypertension was described as mean pulmonary artery pressure $>20 \mathrm{mmHg}$ [11]. Pulmonary hypertension patients suffered from dyspnea after minimal or moderate exertion and PHT was attributed to COPD in our patients.

\section{Measurement of pulmonary artery pressures:}

As recommended by the European Society of Cardiology, Doppler echocardiography was used to evaluate mean and systolic pulmonary artery pressures [11]. Doppler interrogation of the tricuspid and pulmonic valves was considered as one of the first steps in evaluation. The pulmonary arterial pressure was calculated using the measurement of the peak velocity of the tricuspid regurgitation flow by continuous Doppler wave, pulsed Doppler recordings of time-to-peak velocity curves of pulmonary artery blood flow and right ventricular outflow tract [14]. 
Biochemical analyses: Blood samples were obtained by peripheral venipuncture early in the morning (06:00-08:00 A.M) from antecubital veins after an overnight fasting period. Before the blood samples were taken for hematological and biochemical analyses, patients were kept resting for 30 minutes. Strenuous exercise and smoking have been avoided the day before the collection of blood samples. Separation of sera from blood cells was accomplished by centrifugation at $4000 \mathrm{~g}$ for 15 minutes. Serum samples were maintained at $-80^{\circ} \mathrm{C}$ until measurement. The assignment of CAT activity was made with respect to the Aeibi method in which $\mathrm{H}_{2} \mathrm{O}_{2}$ was used as a substrate [12]. Measurement of MDA was carried out spectrophotometrically based on the reaction between thiobarbiturate and MDA using Thiobarbituric acid-reacting substance (TBARS) assay. Glutathione levels were determined with respect to the methodology described by Beutler et al [13]. Analyses were made using a Shimadzu UV-1800 UV-VIS spectrophotometer device (Shimadzu Corp., Kyoto, Japan).

Statistical Analyses: Analysis of data was made via the Statistical Package for Social Sciences (SPSS) software version 20 for Windows (SPSS Inc, Chicago, IL). The conformability of the univariate data to the normal distribution was examined using the Kolmogorov-Smirnov test. Parameters that display normal distribution were evaluated with parametric methods, whereas variables without a normal distribution were analyzed via non-parametric methods. For variables with normal distribution, the correlation was assessed with the Pearson Correlation test. In case variables did not display normal distribution, Spearman's rho test was utilized. A one-way ANOVA test was used to evaluate the differences among groups. Quantitative variables were expressed as either mean \pm standard deviation or median-interquartile range. The confidence interval was set at $95 \%$ and a difference was termed as statistically significant if $\mathrm{p}$ $<0.05$.

\section{Results}

The average age for the whole population was $57.68 \pm 7.60$ and COPD patients were significantly older than the control group ( $59.83 \pm 8.03$ vs 55.53 $\pm 6.60, \mathrm{p}=0.027)$. No significant difference was noted between COPD patients with and without PHT regarding age $(\mathrm{p}=0.786)$.

Analysis of data has shown that levels of MDA $(\mathrm{p}<0.001)$ and CRP $(\mathrm{p}<0.001)$ were increased in COPD patients. In contrary, oxygen saturation $(\mathrm{p}<0.001)$, ejection fraction $(\mathrm{p}=0.002)$, serum levels of GSH $(p<0.001)$ and CAT $(p<0.001)$ were significantly lower in COPD patients (Table 1).
(Table 2) demonstrates a comparative presentation of all variables under investigation in COPD group with PHT $(n=18$, $60 \%)$ and without PHT ( $n=12,40 \%)$. Notably, 2 subgroups of COPD did not exhibit any remarkable difference in terms of oxidative stress indicators MDA, GSH, and CAT activity.

Results of correlation analysis in the whole group are plotted in (Table 3). Malondialdehyde was inversely correlated with oxygen saturation and ejection fraction. Serum GSH levels were found to be inversely correlated with MDA levels. The inverse correlations were detected between CRP and ejection fraction as well as between D-dimer and oxygen saturation. 


\begin{tabular}{|l|l|l|l|}
\hline & Control & COPD & $p$ Value \\
\hline GSH $(\mathrm{U} / \mathrm{ml})$ & $0.159 \pm 0.47$ & $0.024 \pm 0.013$ & $<0.001^{\star}$ \\
\hline $\mathrm{MDA}(\mathrm{nmol} / \mathrm{ml})$ & $0.179 \pm 0.057$ & $0.871 \pm 0.265$ & $<0.001^{\star}$ \\
\hline $\mathrm{Hb}(\mathrm{g} / \mathrm{dl})$ & $14.76 \pm 1.50$ & $15.42 \pm 1.75$ & 0.124 \\
\hline $\mathrm{Hct}(\%)$ & $44.38 \pm 4.51$ & $48.06 \pm 6.09$ & $0.010^{*}$ \\
\hline $\mathrm{LDL}(\mathrm{mg} / \mathrm{dL})$ & $109.40 \pm 31.08$ & $98.97 \pm 38.22$ & 0.251 \\
\hline $\mathrm{TG}(\mathrm{mg} / \mathrm{dL})$ & $116.77 \pm 81.12$ & $126.63 \pm 56.92$ & 0.588 \\
\hline $\mathrm{HDL}(\mathrm{mg} / \mathrm{dL})$ & $45.77 \pm 10.80$ & $39.20 \pm 7.50$ & $0.009^{*}$ \\
\hline $\mathrm{Cholesterol}(\mathrm{mg} / \mathrm{dL})$ & $178.73 \pm 41.25$ & $163.23 \pm 45.67$ & 0.173 \\
\hline $\mathrm{D}-\mathrm{dimer}(\mu \mathrm{g} / \mathrm{l})$ & $152.93 \pm 95.29$ & $228.70 \pm 216.63$ & 0.087 \\
\hline EF $(\%)$ & $64.07 \pm 4.81$ & $60.27 \pm 4.07$ & $0.002^{\star}$ \\
\hline $\mathrm{O}_{2}$ saturation $(\%)$ & $96.97 \pm 1.67$ & $83.60 \pm 5.44$ & $<0.001^{\star}$ \\
\hline $\mathrm{CAT} \dagger(\mathrm{U} / \mathrm{ml})$ & $0.002-0.002$ & $0.0004-0.000$ & $<0.001^{\star}$ \\
\hline $\mathrm{CRP} \dagger(\mathrm{mg} / \mathrm{l})$ & $0.400-0.388$ & $0.670-0.648$ & $<0.001^{\star}$ \\
\hline
\end{tabular}

Table 1. Overview of parameters under investigation in COPD patients and the control group.

(Abbreviations: COPD: chronic obstructive pulmonary disease; GSH: glutathione peroxidase; MDA: malondialdehyde; Hb: hemoglobin; Hct: hematocrit; LDL: low-density lipoproteins; TG: triglycerides; HDL: high-density lipoproteins; EF: ejection fraction; CAT: catalase; CRP: C-reactive protein; *: statistically significant; $\dagger$ : expressed as median-interquartile

\begin{tabular}{|l|l|l|l|}
\hline & PHT (-) & PHT $(+)$ & $p$ Value \\
\hline GSH $(\mathrm{U} / \mathrm{ml})$ & $0.023-0.008$ & $0.022-0.015$ & 0.385 \\
\hline $\mathrm{MDA}(\mathrm{nmol} / \mathrm{ml})$ & $0.769-0.613$ & $0.801-0.265$ & 0.362 \\
\hline $\mathrm{Hb}(\mathrm{g} / \mathrm{dl})$ & $15.35-1.10$ & $15.80 \pm 3.35$ & 0.539 \\
\hline $\mathrm{Hct}(\%)$ & $47.45-5.92$ & $49.45-11.80$ & 0.672 \\
\hline $\mathrm{LDL}(\mathrm{mg} / \mathrm{dL})$ & $124.00-39.50$ & $85.50-62.25$ & $0.046^{*}$ \\
\hline $\mathrm{TG}(\mathrm{mg} / \mathrm{dL})$ & $152.00-119.75$ & $105.00-64.50$ & $0.038^{*}$ \\
\hline $\mathrm{HDL}(\mathrm{mg} / \mathrm{dL})$ & $40.50-10.25$ & $43.00-12.50$ & 0.298 \\
\hline $\mathrm{Cholesterol} \mathrm{(mg/dL)}$ & $193.00-52.25$ & $160.00-53.25$ & 0.117 \\
\hline $\mathrm{D}-\mathrm{dimer}(\mu \mathrm{g} / \mathrm{l})$ & $167.00-156.00$ & $140.00-213.00$ & 0.882 \\
\hline EF $(\%)$ & $60.00-7.50$ & $60.00-6.25$ & 0.687 \\
\hline O saturation $(\%)$ & $86.00-5.25$ & $82.00-9.00$ & 0.089 \\
\hline CAT $(\mathrm{U} / \mathrm{ml})$ & $0.0004-0.0003$ & $0.0004-0.0004$ & 0.866 \\
\hline CRP (mg/l) & $0.67-0.66$ & $0.67-0.70$ & 0.799 \\
\hline
\end{tabular}

Table 2. Comparison of COPD patients with and without PHT $\dagger$.

(Abbreviations: COPD: chronic obstructive pulmonary disease; GSH: glutathione peroxidase; MDA: malondialdehyde; Hb: hemoglobin; Hct: hematocrit; LDL: low-density lipoproteins; TG: triglycerides; HDL: high-density lipoproteins; EF: ejection fraction; CAT: catalase; CRP: C-reactive protein; ${ }^{*}$ : statistically significant)

$\dagger$ : All data have been expressed as a median-interquartile range. 


\begin{tabular}{|c|c|c|c|}
\hline Variable & Correlate & $p$ Value & $\mathbf{r}$ \\
\hline Age & MDA & 0.032 & 0.282 \\
\hline \multirow{2}{*}{ GSH } & MDA & 0.001 & -0.418 \\
\hline & TG & 0.004 & 0.375 \\
\hline \multirow{2}{*}{ MDA } & $\mathrm{EF}$ & 0.025 & -0.293 \\
\hline & $\mathrm{O}_{2}$ saturation & $<0.001$ & -0.536 \\
\hline Hct & HDL & 0.019 & -0.307 \\
\hline \multirow{2}{*}{ LDL } & TG & 0.005 & 0.361 \\
\hline & Cholesterol & $<0.001$ & -0.536 \\
\hline \multirow{2}{*}{ TG } & $\mathrm{HDL}$ & 0.032 & -0.282 \\
\hline & Cholesterol & $<0.001$ & 0.514 \\
\hline Cholesterol & CRP & 0.012 & -0.327 \\
\hline CRP & $\mathrm{EF}$ & 0.002 & -0.392 \\
\hline D-dimer & $\mathrm{O}_{2}$ saturation & 0.022 & -0.301 \\
\hline
\end{tabular}

Table 3. Correlation of biochemical, inflammatory and cardiorespiratory variables in the whole study population.

(Abbreviations: GSH: glutathione peroxidase; MDA: malondialdehyde; Hct: hematocrit; LDL: low-density lipoproteins; TG: triglycerides; HDL: high-density lipoproteins; EF: ejection fraction; CRP: C-reactive protein; ${ }^{*}$ statistically significant)

\section{Discussion}

In this study, we aimed to investigate whether serum levels of oxidative stress markers were altered in COPD patients with and without PHT. Furthermore, we tested if any correlations existed between these hematological, biochemical or clinical parameters. Our results have shown that oxidative stress markers are altered in COPD; however, no noteworthy differences could be determined between COPD patients with and without PHT.

Oxidation is a critical factor in the pathogenesis and pathophysiology of COPD [1,3]. Oxidative stress may have a deleterious impact on the prognosis and if so, adequate supplementation with antioxidants may be beneficial for COPD patients [2].

Higher levels of CRP and MDA in COPD patients remind an inflammatory basis linked with oxidative stress in the pathophysiology of COPD. In accordance with this data, we detected lower levels of CAT and GSH in our series. From this point of view, our results are similar to those of Joppa et al. [5]. Joppa et al. reported that there are decreased levels of GSH in COPD patients with PHT this group and this may be due to the fact that these peptides serve both in pathophysiology and pulmonary vascular physiology [5]. However, in the present study, no differences could be found COPD patients with and without PHT regarding oxidative stress indicators. Controversially, Cracowski et al. have reported increased markers of lipid peroxidation (such as MDA) in severe PHT patients [15]. They stated that oxidative stress is increased in patients with PHT. The results of the present study do not support these findings who have reported a decreased antioxidant enzyme activity in the lungs of patients with severe PHT [15].

Attributed to the complex interaction of molecules and enzymes in inflammatory and oxidative cascade, some of our findings are contradictory to some publications in the literature. For instance, Liu et al. also noted increased expression of GSH in patients with PHT, highlighting its critical role in vascular homeostasis [16]. Therefore, these molecules may have multiple functions and predominating functions may vary under different physiological circumstances. Doubtlessly, deficiency of GSH is consistent with increased oxidative stress and the eventual injury is supposed to be a crucial step in the subsequent steps of pathogenesis [8].

It must be kept in mind that reactive oxygen species in pulmonary circulation are balanced by the counter activity of antioxidant mechanisms. Levels of MDA have been associated with the severity of pulmonary functions in COPD [17] and a direct relationship was established between glutathione peroxidase levels and forced expiratory volume in one second [3]. In the lung, GSH and CAT serve as hydrogen peroxide-scavenging enzymes, and in agreement with the data in the literature, we came across a lower GSH activity in COPD patients [4].

With respect to the potential role of lipid peroxidation in the progression of COPD, an association between oxidative 
stress and PHT may be remembered. Nevertheless, the results of the present study seem not to support such an association. Effective therapeutic antioxidant measures are needed to control COPD, PHT and other respiratory diseases

In the present study, serum levels of malondialdehyde and glutathione, and catalase activity did not differ between COPD patients with and without PHT. Although the reason for this insignificance could not be explained, perhaps not the pulmonary artery pressure but the evaluation of right ventricle (RV) discriminate the difference in oxidative stress, measured by the size of the RV, TAPSE, FAC, S wave or even cardiac output (measured on catheterization). This theory may be studied in the near future.

Herein, the diagnosis of pulmonary hypertension was established by echocardiographic measurements. Recently, Doppler echocardiography has gained popularity in the last 2 decades for non-invasive estimation of PHT [18]. However, in patients with COPD, echocardiography has been reported to perform variable in the assessment of PHT [19]. The diagnosis of PHT constitutes the major limitation of the present study. A small sample size and cross-sectional design constitute other limitations of this study. Single measurements are prone to be influenced by many conditions and may not always reflect the actual state. Moreover, lack of control of genetic, social, ethnic and environmental factors may restrict the extrapolation of our results to larger populations. In addition, PHT is not a standard and uniform entity and it may present in various clinical forms. Finally, there is no match of sex and age between COPD patients and the control group. Limitations of the TBARS method should also be expressed. TBARS tests rarely measure the free MDA content of the lipid system but rather measure MDA generated by decomposition of lipid peroxides during the acid-heating stage of the test [20]. In addition, several other compounds, including sugars, amino acids, and bilirubin are also reactive toward TBA. The sensitivity of the assay can be increased by combining it with HPLC to separate such compounds prior to acid heating.

To conclude, the results of the current study support the roles of inflammation and oxidative stress in COPD, but no remarkable alterations in oxidative stress indicators could be identified in COPD patients with PHT. Therefore, further trials on larger series are required to elucidate the role of oxidative stress and to assess the effects of antioxidants in COPD and PHT. 


\section{References}

1. Lee JS, Shin JH, Hwang JH, Baek JE, Choi BS (2014) Malondialdehyde and 3-nitrotyrosine in exhaled breath condensate in retired elderly coal miners with chronic obstructive pulmonary disease. Saf Health Work 5: 91-96.

2. Ben Moussa S, Sfaxi I, Tabka Z, Ben Saad H, Rouatbi S (2014)

Oxidative stress and lung function profiles of male smokers free from COPD compared to those with COPD: a case-control study. Libyan J Med 9: 23873.

3. Ford ES, Li C, Cunningham TJ, Croft JB (2014) Associations between antioxidants and all-cause mortality among US adults with obstructive lung function. Br J Nutr 112: 1662-1673.

4. Domej W, Oettl K, Renner W (2014) Oxidative stress and free radicals in COPD - implications and relevance for treatment. Int J Chron Obstruct Pulmon Dis 9: 1207-1224.

5. Joppa P, Petrásová D, Stancák B, Dorková Z, Tkácová R (2007) Oxidative stress in patients with COPD and pulmonary hypertension. Wien Klin Wochenschr 119: 428-434.

6. Hurdman J, Condliffe R, Elliot CA, Swift A, Rajaram S, Davies C, et al. (2013) Pulmonary hypertension in COPD: results from the ASPIRE registry. Eur Respir J 41: 1292-1301.

7. Held M1, Jany BH (2013) Pulmonary hypertension in COPD. Respir Care 58: e86-91.

8. Reis GS, Augusto VS, Silveira AP, Jordão AA Jr, et al. (2013) Oxidative-stress biomarkers in patients with pulmonary hypertension. Pulm Circ 3: 856-861.

9. Bowers R, Cool C, Murphy RC, Tuder RM, Hopken MW, Flores SC, et al. (2004) Oxidative stress in severe pulmonary hypertension. Am J Respir Crit Care Med 169: 764-769.

10. West JB (2013) GOLD Executive Summary. Am J Respir Crit Care Med 188: 1366-1367.

11. Galiè N, Hoeper MM, Humbert M, Torbicki A, Vachiery JL, Barbera JA, et al. (2009) ESC Committee for Practice Guidelines (CPG). Guidelines for the diagnosis and treatment of pulmonary hypertension: the Task Force for the Diagnosis and Treatment of Pulmonary Hypertension of the European Society of Cardiology (ESC) and the European Respiratory Society (ERS), endorsed by the International Society of Heart and Lung Transplantation (ISHLT). Eur Heart J 30: 2493-2537.

12. Aebi H (1984) Catalase in vitro. Methods Enzymol 105: 121126.

13. Beutler E, Gelbart T (1985) Plasma glutathione in health and in patients with malignant disease. J Lab Clin Med 105: 581-584. 14. Barst RJ, McGoon M, Torbicki A, Sitbon O, Krowka MJ, Olschewski H, et al. (2004) Diagnosis and differential assessment of pulmonary arterial hypertension. J Am Coll Cardiol 43: 40S-47S. 15. Cracowski JL, Cracowski C, Bessard G, Pepin JL, Bessard J,
Schwebel C, et al. (2001) Increased lipid peroxidation in patients with pulmonary hypertension. Am J Respir Crit Care Med 164: 1038-1042.

16. Liu J, Thomas PS (2005) Exhaled breath condensate as a method of sampling airway nitric oxide and other markers of inflammation. Med Sci Monit 11: M53-M62.

17. Ochs-Balcom HM, Grant BJ, Muti P, Sempos CT, Freudenheim JL, Browne RW, et al. (2006) Anti-oxidants, oxidative stress and pulmonary function in individuals diagnosed with asthma or COPD. Eur J Clin Nutr 60: 991-999.

18. Nobre C, Mesquita D, Thomas B (2013) Echocardiography in pulmonary hypertension. Chest 144: 1426.

19. Iyer AS, Wells JM, Vishin S, Bhatt SP, Wille KM, Dransfield MT (2014) CT scan-measured pulmonary artery to aorta ratio and echocardiography for detecting pulmonary hypertension in severe COPD. Chest 145: 824-832.

20. Gutteridge JMC (1986) Aspects to consider when detecting and measuring lipid peroxidation. Free Radic Res Commun 1: 173-184.

\footnotetext{
Submit your manuscript to a JScholar journal and benefit from:

๑ Convenient online submission

ฯ Rigorous peer review

I Immediate publication on acceptance

ๆ Open access: articles freely available online

ब High visibility within the field

ब Better discount for your subsequent articles Submit your manuscript at http://www.jscholaronline.org/submit-manuscript.php
} 